\title{
Male and Couple Fertility Impairment due to HPV-DNA Sperm Infection: Update on Molecular Mechanism and Clinical Impact-Systematic Review
}

\author{
Salvatore Gizzo, ${ }^{1}$ Bruno Ferrari, ${ }^{2}$ Marco Noventa, ${ }^{1,3}$ Emanuele Ferrari, ${ }^{2}$ \\ Tito Silvio Patrelli, ${ }^{2}$ Michele Gangemi, ${ }^{1}$ and Giovanni Battista Nardelli ${ }^{1}$ \\ ${ }^{1}$ Department of Woman and Child Health, University of Padua, 35128 Padua, Italy \\ ${ }^{2}$ Department of Surgical Sciences, University of Parma, 43100 Parma, Italy \\ ${ }^{3}$ Dipartimento di Salute della Donna e del Bambino, U.O.C. di Ginecologia e Ostetricia, Via Giustiniani 3, 35128 Padova, Italy
}

Correspondence should be addressed to Marco Noventa; marco.noventa@gmail.com

Received 4 January 2014; Accepted 10 February 2014; Published 25 March 2014

Academic Editor: Irma Virant-Klun

Copyright (c) 2014 Salvatore Gizzo et al. This is an open access article distributed under the Creative Commons Attribution License, which permits unrestricted use, distribution, and reproduction in any medium, provided the original work is properly cited.

Recent evidences identify Human Papillomavirus (HPV) sperm infection as a possible cause of male and couple infertility. It acts through different mechanisms at various steps of human conception and early gestational development. We performed a systematic review to assess the role of HPV semen infection on male and couple infertility. Analysis of available and eligible data does not permit us to fund clear evidences about clinical impact of HPV infection on fertility, although sperm parameters impairment is the most widely recognized effect. Regarding biomolecular implications, the available data are often conflicting. More studies are required to define the role of HPV sperm infection in clinical practice. The great majority of evidences are obtained by in vitro studies and this fact represents a limitation for the clinical management of HPVDNA sperm infection. Understanding the biological significance of HPV-DNA semen infection could permit us to explain most of the idiopathic male and couple infertility, leading to a better management of infertile men and a better timing for sperm banking storage before ART cycles.

\section{Introduction}

Human Papillomavirus (HPV) infection is one of the main sexually transmitted diseases worldwide [1]. The most important clinical consequence of HPV infection is cervical cancer which remains one of the leading causes of cancer-related mortality in young and older women [2-5].

HPV infection is also related to anogenital warts and different neoplasia, such as vaginal, vulvar, penile, anal, oral cavity, head, and neck cancers $[1,4,6]$. Considering male and female, the overall prevalence of HPV infection is about $40 \%$ of population, with differences based on the HPV type and the anatomical site of infection [7].

Great progresses have been reached in understanding the pathologic mechanisms of HPV infection. Both effective screening programs (pap smears, HPV-DNA testing) and interventions (HPV vaccination) have been developed in order to reduce HPV related disease in women [8-12].

Nevertheless few data are available on male infection $[1$, 13-15].

Recent findings underlined the role of HPV semen infection in male and couple infertility, focusing mainly on its high prevalence among 18-40 years old men [16, 17].

The exact localization of HPV in the spermatozoa is not well defined, even if recent studies demonstrated that the virus binds two distinct sites along the equator of the spermatozoa's head $[18,19]$.

Many Authors hypothesized that HPV can modify spermatic parameters causing sperm motility reduction, seminal $\mathrm{pH}$ alterations, and spermatozoa DNA fragmentation $[18,20-$ 22]. 
Although in vitro studies demonstrated that spermatozoa can carry HPV-DNA and transfer it to oocytes, it is still not clear if in vivo the $\mathrm{HPV}$-infected sperm is able to fertilize oocyte and to transfer the viral genome [17, 2325]. These uncertainties extend to the following steps of conception because it is not clear if the infected oocytes are able to generate normal embryo and if the infection itself could interfere with implantation and subsequent pregnancy development [17].

The aim of this review is to investigate the implications of HPV sperm infection on male and couple infertility, analyzing the clinical impact on early pregnancy development and pregnancy loss, the paradigmatic spermatic alterations, the sperm immunological modifications, and the spermatozoa HPV-related molecular changes. Finally we analysed the effect of HPV-infected sperm on fertilized oocyte, blastocyst implantation, and pregnancy development. We will also discuss the available diagnostic and therapeutic strategies, in terms of feasibility and clinical efficacy trying to make some considerations on sperm banking before ART cycles.

\section{Data Sources}

A Literature analysis was performed on the electronic databases Medline, Embase, ScienceDirect and the Cochrane Library, considering papers published in the time interval from 1994 to 2013.

We looked for randomized trials, observational and retrospective studies, original works, and review articles having topics as the relation between male HPV sperm infection, seminal modifications, effects on fertilized oocytes, association to apoptosis, early miscarriages, and implications on male and couple reproductive outcomes.

Key-terms included "HPV sperm infection," "male infertility and HPV," "sperm parameters and HPV," "HPV infected sperm and fertilization," "HPV and fertility outcome," and "HPV and blastocyst apoptosis." An accurate analysis of the references of the main works was successively performed.

We considered data from eligible studies separately, according to different topics, "clinical impact of HPV infection and fertility outcomes," "HPV-related spermatic modifications and their impact on fertility," and "ability of infected semen to vehicle exogenous HPV-DNA and its impact upon ongoing pregnancy."

\section{Methods}

We evaluated the clinical significance of genital HPV-DNA presence in male, female, and couple in relation to placental infection (both at term and preterm) and spontaneous miscarriage (Table 1).

The seminal parameters were defined according to $\mathrm{WHO}$ laboratory manual for examination and processing of human semen [5] and Hamilton Thorn motility analyser [42].

We analysed both clinical and experimental works which focused on the ability of human sperm to vehicle HPV-DNA infection into oocytes and subsequent implications (Table 2).
HPV-DNA detection in various sample tissues was based on polymerase chain reaction (PCR) and fluorescence in situ hybridization (FISH) techniques.

The sperm washing techniques reported in the studies were two-layer isolate colloid wash, test-yolk buffer procedures, swim-up procedure, modified swim up with enzymatic treatment (Heparinase-III), and discontinuous Ficoll gradients. We included also studies which used DNA disc chip assay, Comet assay, and Cell Death Detection ELISA in order to detect HPV-related cells apoptosis.

Finally the hamster egg-human sperm penetration test (HEPT) has been used in some works in order to assess the ability of HPV-infected sperm to fertilize and to transfer viral genome into the oocytes.

\section{Results}

According to our selection criteria, only 23 works had eligible results for the aim of the review.

4.1. Clinical Impact of HPV Infection and Fertility Outcomes. Only 5 papers focused on this topic (Table 1). Hermonat et al. in 1997 firstly performed a study evaluating the presence of HPV-DNA in 25 spontaneous early miscarriages versus 15 voluntary abortions. 15 of the 25 spontaneous samples (60\%) were found to be positive for HPV E6/E7 sequences versus only 3 of the 15 elective samples (20\%). Semiquantitative analysis showed that the HPV detection was six-fold higher in the spontaneous abortions compared to elective ones $(P<$ $0.01)$ [26].

Matovina et al. analyzed the products of conception from early miscarriage detecting a HPV 16-18 incidence of 7.4\% [27].

Dana et al. focused on the exposure to HPV type $6 / 11 / 16 / 18$ during pregnancy and found a spontaneous abortion rate of $6.9 \%$, a prevalence of major birth defects of $2.2 \%$ and fetal death rate of $1.5 \%$ [28].

In 2010 Skoczynski et al. compared the prevalence of HPV infection in placentas from term deliveries and spontaneous abortions. The comparison between the two groups showed no differences in terms of HPV-DNA detection since it was found in $24.4 \%$ of placentas at term $(12.8 \%$ of HPV $16 / 18$ types) and in $17.7 \%$ of miscarriages (11.8\% of HPV 16/18) [p:n.s.] [29].

Perino et al. in a cohort of 199 couples undergoing ARTs reported a prevalence of 9.5\% (19/199) for HPV male infection, $17.5 \%$ (35/199) for female infection, and $4.5 \%$ (9/199) for both partners infection. After ARTs, miscarriage rate had considerably higher results in couples where a single or both partners were HPV carriers compared to noninfected couples. The comparison between the two groups resulted in $66.7 \%$ versus $15 \%$ in case of male infection $(P<0.01)$, $40 \%$ versus $13.7 \%$ in case of female infection, and $100 \%$ versus $15.9 \%$ in case of both partners infections $(P<0.001)$ [30].

The main limitations of all these studies are certainly related to the small sample size. Moreover, all the considered studies were retrospective or cross-sectional since the only perspective one was performed by Perino et al. [30]. 


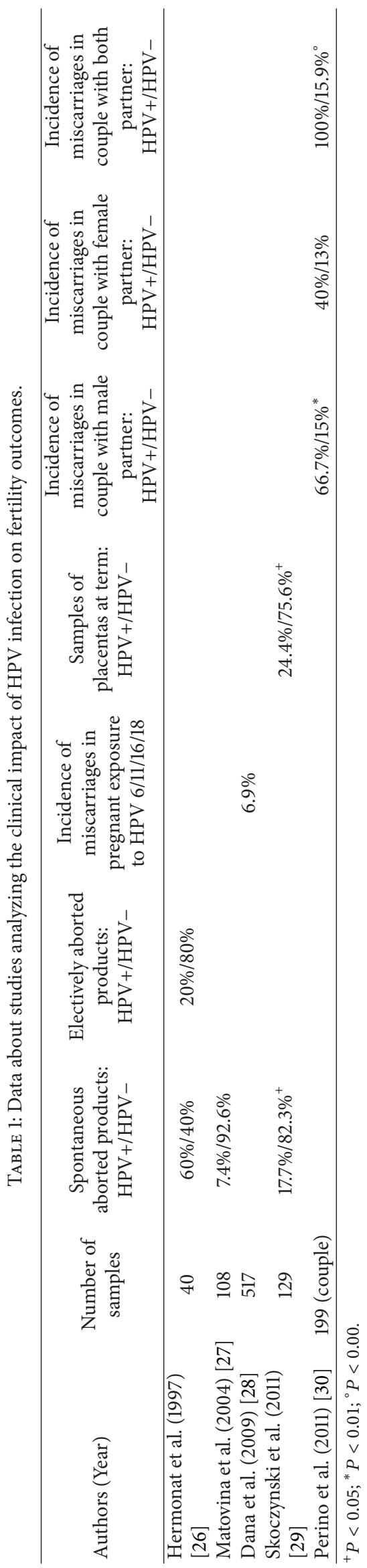




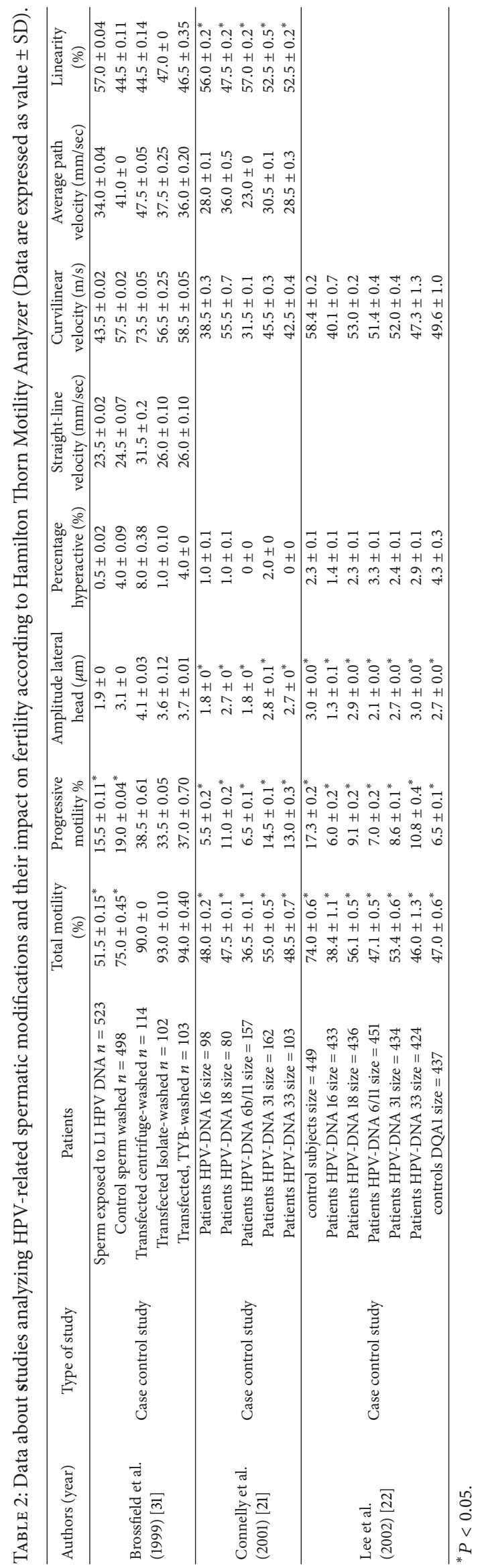


4.2. HPV-Related Spermatic Modifications and Their Impact on Fertility. Only 9 eligible studies focused on this topic (Tables 2 and 3).

Both Brossfield et al. and Connelly et al. analysed the spermatic effects of HPV infection, showing that sperm cells transfected with exogenous HPV-DNA had higher percentages of total motility and progression compared to the untreated controls $(P<0.05)$ [21, 31].

On the contrary, Lee et al. demonstrated that sperm motility was reduced after HPV E6-E7 fragments expression. The percentages of progressive motility were lower in sperm exposed to all the HPV-DNA genotypes (except for the 33); the amplitude of lateral head displacement was decreased after exposure to (high-risk) hrHPV-DNA type 16 and all (low-risk) lrHPV-DNA $(P<0.05)$ [22].

In 2004 Rintala et al. analysed 65 sperm donors, $15.5 \%$ results were positive for hrHPV-DNA and $17 \%$ positive for lrHPV-DNA. They reported no HPV-related effects on sperm motility and concentration, except for $\mathrm{pH}$ changes [20].

Foresta et al. evaluated 200 male sperm donors observing that sperm parameters results were similar between the two groups except for the sperm motility which was significantly reduced in HPV-DNA infected men $(P<0.05)$ [18].

In the same year, Foresta et al. showed that HPV-DNA was frequently detected in exfoliated epithelial cells of lower genital tract [77.8-100\%], while spermatozoa infection was detected in $72 \%$ of infertile men. Despite the mean sperm motility results significantly reduced in all infected men compared to noninfected ones $(P<0.05)$, all the remaining parameters were similar between infected or noninfected patients. A reduction of sperm concentration was registered in case of infertility, independently from HPV-DNA infection $(P<0.05)[32]$.

Considering sperm-washing procedure (in order to remove HPV-DNA from the sperm surface) Brossfield et al. showed that all the applied techniques did not positively influence the spermatic HPV-related motility and that, between them, the centrifuge wash technique results were the best ones [31].

On the other hand Foresta et al. observed that only Ficoll and swim-up procedure were useful to reduce the infection in $30 \%$ and $26 \%$ of sample, respectively $(P<0.01)$ [33].

Garolla et al. demonstrated the negative effects of HPV infection reporting that only progressive sperm motility was significantly reduced in infected semen samples $(P<$ $0.05)$. Authors reported that direct swim-up reduces up to $24 \%$ the HPV sperm infection, while modified swim-up is able to remove completely HPV-DNA from spermatozoa $(P<0.01)$. Anyway, Authors underlined how modified swim-up results were responsible for a slight decrease of sperm motility, viability, and DNA integrity [17]. The same author in 2013, using the sperm-Mar test to detect antisperm antibodies (ASA), demonstrated that infertile men showed more frequently ASA than fertile ones $(P<0.01)$. The evidences that men with HPV-DNA infection associated to positive sperm-Mar test are affected by 24 months lower spermatozoa motility than negative ones lead the authors to propose the positive sperm-Mar test as predictor tool for future progressive spermatozoa' motility [34].
4.3. Infected Semen Ability to Transmit Exogenous HPVDNA and Its Impact upon ongoing Pregnancy. Only 11 studies analysed sperm ability to carry exogenous HPV-DNA into oocytes and viral genome into the blastocysts and its impact in terms of fertility, implantation, and embryonic effects (Table 4).

Even if Chan et al. in 1994 showed that human sperm could be infected by HPV-DNA, Lai et al. in 1996 first assumed that spermatozoa could act like a vector for HPV transmission to sexual partners and to foetus through fertilized eggs [35, 43].

Later, both Connelly et al. and Lee et al. demonstrated increased apoptotic phenomena in sperm cells exposed to E6/E7 genes of HPV-DNA types 16 and $18(P<0.05)$ [21, 22].

Chan et al. demonstrated in a mouse experimental model the ability of HPV-DNA infected sperm to transmit its genome to blastocysts [37]. Cabrera et al. demonstrated the presence of HPV-DNA in both the inner cell mass and trophoblastic cells of murine infected blastocysts [23].

Foresta et al., using the hamster egg-human sperm penetration test (HEPT), demonstrated the sperm ability to transfer both the capsid protein L1 and E6/E7 viral genes to oocytes with a subsequent gene expression by transfected blastocysts [25].

Calinisan et al., transfecting blastocysts with the E6-E7 region of types $16,18,31$, and 33 , detected the presence of DNA fragmentation only in the subgroup of blastocysts infected by HPV-DNA type $16(P<0.05)$. The ability of HPVDNA type 16 to induce DNA fragmentation and subsequent trophoblastic death was also confirmed by You et al. in 2002 [38].

Hennemberg et al. confirmed the direct HPV16 inhibitory effect on blastocysts growth only at the two-cell embryo stages but not on the $4-8$ cells ones [40].

Gomez et al. showed that the apoptosis rate in transfected trophoblastic cells was 3-fold (2.4-3.7) and 5.8-fold (5.6-5.9) greater at 3 and 12 days, respectively, if compared to negative controls $(P<0.01)$. Simultaneously, authors reported that the invasion ability of transfected trophoblast progressively decreased from day 3 to day $15(25.2-57.6 \%$ lower than negative controls $)(P<0.001)[41]$.

The limitations of all these studies were certainly related to the artificial conditions linked to the in vitro experiments and murine models which may not reflect the real in vivo human situations. All these data should be validated by large in vivo perspective studies but unfortunately the literature lacks information about this field because of ethical policies concerning human reproduction experiments.

\section{Discussion}

It is widely proven that sexually transmitted infections (STD) represent a possible cause of male infertility since they can induce urethral stickiness, epididymis inflammation, and orchitis up to testicular failure [44]. The impairment of sperm motility and DNA integrity through an autoimmune mechanism could result in obstetric complications such as early miscarriages and preterm deliveries [27, 45]. 


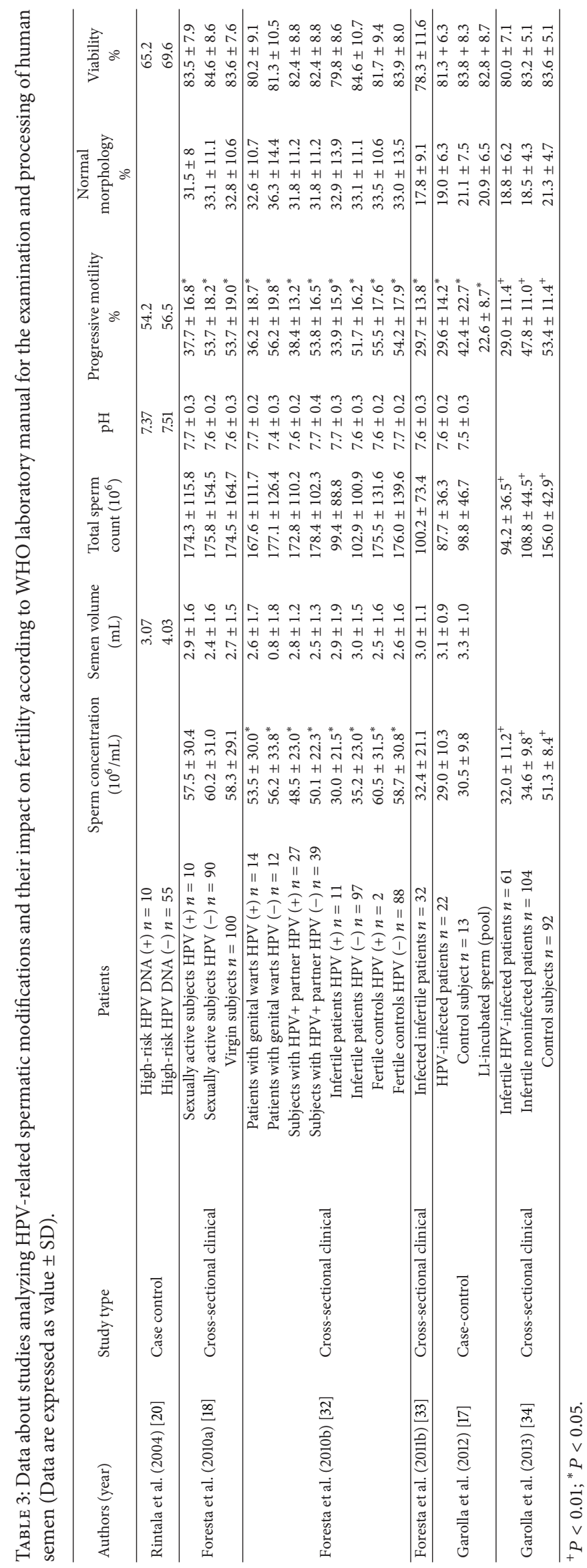




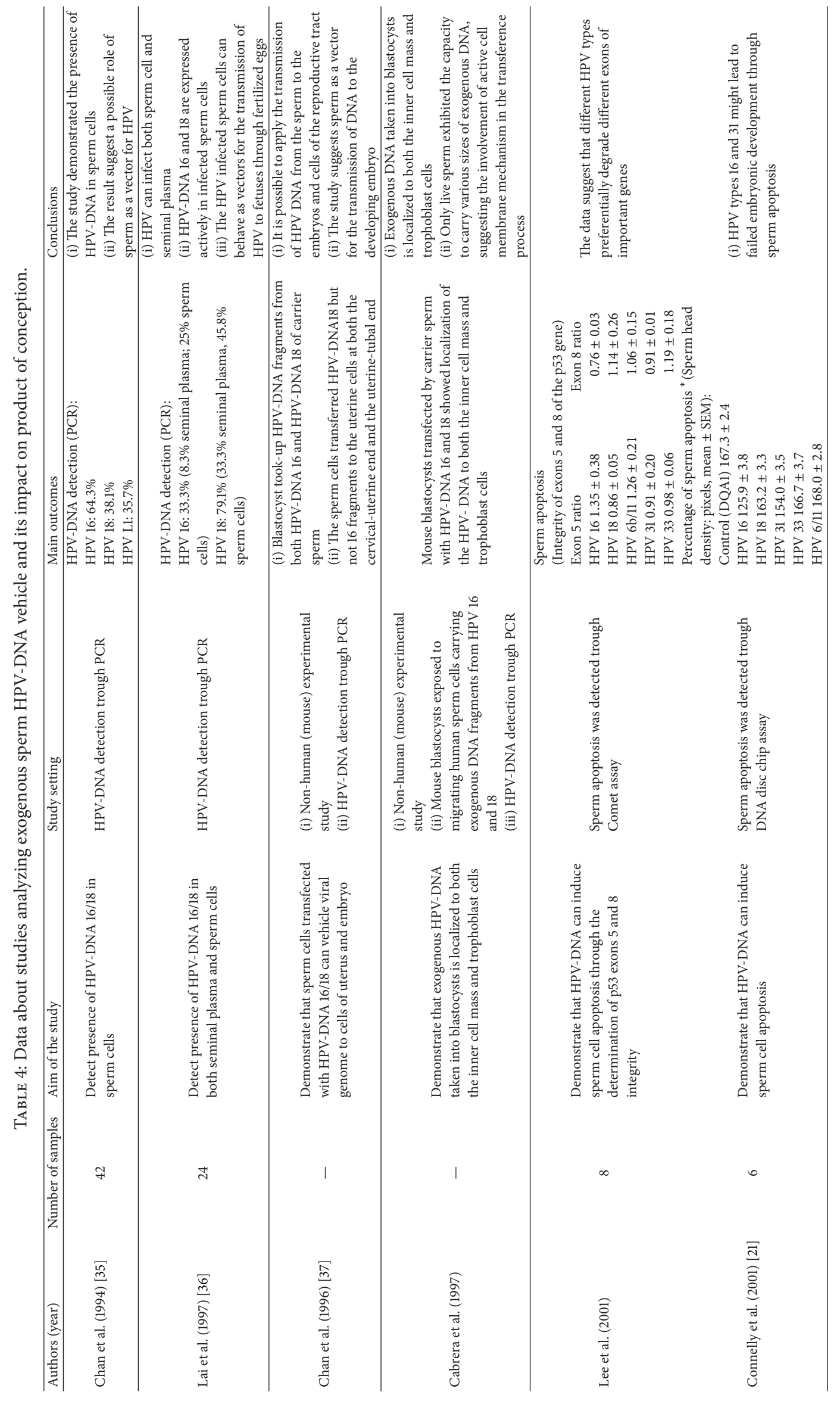




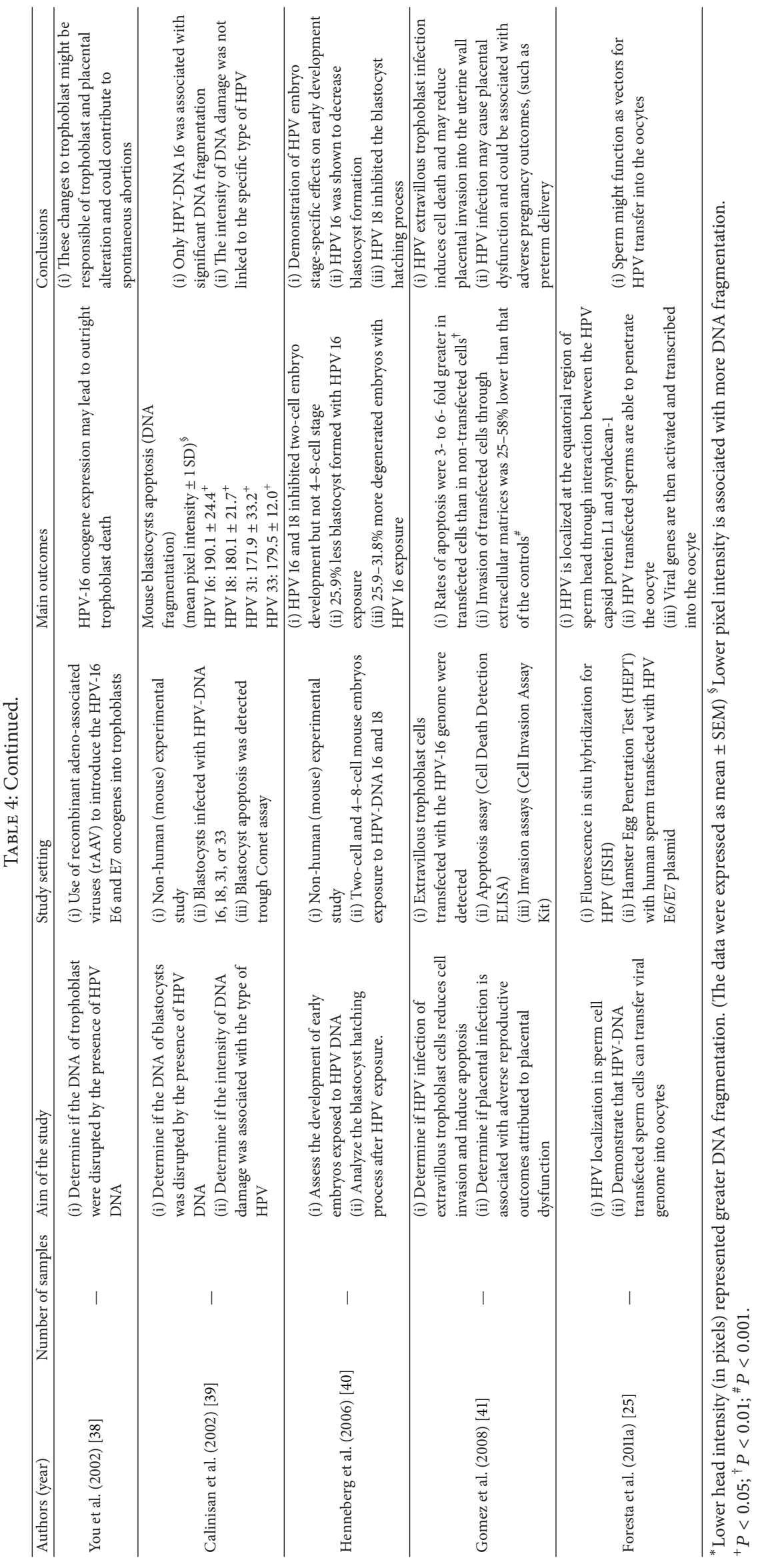


The high prevalence of male HPV detection raised strong interest for its possible consequences on male fertility. HPV infection natural history in men is still mostly unknown. Recent findings suggest that the incidence of genital HPV infection is 38.4 per 1000 male/months (95\% CI 34.3-43.0). The mean duration of male infection results of 7-52 months are (6.80-8.61) for any HPV type and of 12-19 months (7.1618.17) are for hrHPV 16 [46].

5.1. Clinical Impact of HPV Infection and Fertility Outcomes. All in vitro studies showed a negative influence of $\mathrm{HPV}$ upon several aspects of male fertility. However, only few studies investigated the in vivo effects of HPV on human reproduction phases. In 1997 Hermonat et al. reported the correlation between HPV infection and spontaneous miscarriages in first trimester of pregnancy. The higher percentage of HPV-DNA detection, if compared to voluntary abortions, paved the way to consider HPV as one of the possible etiologic agents responsible for early pregnancy loss [26]. This hypothesis was not confirmed by Matovina et al. who found HPV-DNA in only $7.4 \%$ of spontaneous miscarriage specimens. Anyway the authors did not exclude the possibility of HPV-DNA transplacental transfer [27].

Nowadays, in spontaneous conception, the rate of early miscarriages and major birth defects does not seem greater in HPV-exposed couples than in unexposed ones [28, 29]. However, considering in vitro fertilization techniques, this aspect seems to be of crucial importance. The first prospective study on this topic reported a significant pregnancy loss increase in couples undergone ARTs and male partner with semen HPV infection (miscarriage rate of $100 \%$ when both partners results are infected) [30].

So we can conclude that the role of HPV infection in spontaneous abortion is still not clear and its association with adverse pregnancy outcomes is not certainly demonstrated. Further perspective longitudinal studies are necessary to better understand the possible roles of HPV infection in early miscarriage and in other adverse pregnancy outcomes.

However, if the evidences of Perino et al. will be confirmed, a major care on HPV status of couples attempting ART procedures should be necessary. Thus, HPV male vaccination could represent a possible strategy for male fertility preservation and for ART success rate improvement.

\subsection{HPV-Related Spermatic Modifications and Their Impact} on Fertility. The molecular mechanisms by which HPV could impair sperm quality and fertilized oocytes development has been neither completely demonstrated nor clarified.

From our analysis, HPV presence in spermatozoa may be associated with an impairment of sperm parameters. Lai et al. firstly demonstrated that HPV presence in sperm could affect spermatozoa's motility (lower velocity, straight-line velocity, and mean amplitude of lateral head displacement) although it seems to be not statistically significant. A possible association between HPV semen infection and asthenozoospermia has also been described [36]. These findings seem to be partially confirmed even by following studies. Previous works showed that prewashed sperm specimens transfected with L1 HPVDNA fragments had an increased motility and progression but they did not elucidate the possible mechanisms [21, 31]. On the other hand, Lee et al. described a possible association between HPV-DNA presence, sperm motility reduction, and total progression after 24 hours of incubation [22].

The slight motility increase described by Connelly et al. in HPV exposed spermatozoa might be explained considering that the observation was performed after two hours of incubation. This fact suggests that HPV-DNA requires a suitable interval time to determine molecular changes on sperm motility apparatus. The authors reported a mean reduction of lateral head amplitude even if the virus seems not able to decrease oocytes sperm fertilization ability [21].

Rintala et al. in HPV positive semen detected only a $\mathrm{pH}$ change without any impairment of other parameters [20].

For many years semen $\mathrm{pH}$ impairment was considered the most important mechanism explaining fertility decrease due to asymptomatic genital infections, especially the bacterial ones.

In our opinion $\mathrm{pH}$ impairment represents only one of the several factors that may influence male fertility, since the aetiological mechanism could involve many other factors such as mean sperm motility, presence of ASA on the spermatozoa surface, and qualitative semen parameters impairment till asthenozoospermia $[18,32-34]$.

Since several cases of reported idiopathic asthenozoospermia do not present any known risk factor except for the positivity to HPV-DNA, semen washing procedures could represent a way to improve spermatozoa quality before ART procedures $[18,32,34]$.

Starting from these considerations, many studies proposed different semen washing methods to eliminate HPVDNA sperm infection, but all proposed techniques failed in the scope, except for the modified swim-up technique. This last one contemplates enzymatic treatment (Heparinase-III), apparently able to completely remove HPV-DNA from sperm cells although with a deterioration in semen quality.

Nowadays all the available data suggest that the classic procedures cannot eliminate HPV sperm infection and that HPV-DNA semen screening can be considered only as an epidemiological investigation helping to define the best timing (regression of semen infection) to start ART cycles.

It is well demonstrated both in men and women that HPV infection is generally transient and only few patients are subject to persistent infection. Our suggestion to test the sperm for HPV before sperm banking and ART cycles could represent the last option for the clinicians to try to improve the semen quality both for fresh use and for frozen preservation. Certainly, the vaccine option could have useful results for both male and female patients presenting poor reproductive outcomes till the couple infertility [47].

5.3. Ability of Infected Semen to Vehicle Exogenous HPVDNA and Its Impact on Pregnancy Evolution. The HPV-DNA presence in sperm and the related modification induced by the infection seem to play a role in physiopathology of unexplained male infertility. However, in vitro evidences 
showed that HPV infected spermatozoa maintains the ability to fertilize oocytes and to express viral genome in the product of conception. This suspect seems to be confirmed by a higher rate of in vitro blastocysts and trophoblastic HPVrelated apoptosis probably responsible of in vivo fertility rate reduction of infected couples.

Evidences reported by Chan et al. [35], Lai et al. [43], Pérez-Andino et al. [19], and Foresta et al. [25] clarified many aspects of the overall fertility reduction in couple with male HPV infection. The HPV ability to bind the spermatozoa in two distinct sites of the equatorial region through sydecan-1 (a proteoglycan expressed almost exclusively in the equatorial region of sperm head) can explain the sperm ability to carry viral genome into fertilized oocytes and blastocysts $[19,21,22$, 25].

In both experimental murine and in vivo human models it was found that HPV genomes are expressed in fertilized oocytes, blastocysts, and trophoblastic cells [23, 25, 37]. The viral genome could induce cellular changes such as inhibition of zygote growth, decrease in blastocyst formation, inhibition of blastocyst hatching process, and DNA fragmentation and apoptosis, and thus results are often lethal for early embryo development [38-40].

In vitro experiments showed that HPV transfected blastocysts and trophoblastic cells were affected by a reduction in decidua invasion capacity, potentially responsible for a failure of maternal uterine wall invasion by the extravillous trophoblastic cells, subsequent placental dysfunction, and adverse pregnancy outcomes (i.e., early miscarriage) [41].

Nowadays both the exact mechanism and timing through which the HPV infection modifies trophoblastic genes expression and increases cell death have not yet been understood. All the considered studies have important limits which should not be underestimated, linked to the in vitro artificial and experimental conditions. Further validation in in vivo studies could be therefore needed.

5.4. New Insight: HPV Male Vaccination and Sperm Effects. The actual high prevalence of HPV sperm infection represents a large scale problem for the sperm donors banks $[48,49]$.

HPV screening for all semen samples before sperm banking should be considered as a real option of semen storage for future ART cycles.

The rationale is that, when possible, the semen banking should be postponed until HPV infection resolution. The main limitations in this field are linked to the poor knowledge of HPV male infection natural history and of the most adequate interval time to postpone the storage.

Moreover, neither evidences are available about long term effects of previous HPV sperm infection nor which parameters could recover after the infection-linked damages.

In absence of effective and safety sperm washing procedures able to eliminate the infection, HPV male vaccination should be considered as a possible strategy for the prevention of HPV semen impairment and for the improvement of couple fertility outcomes $[4,30,46,48,50,51]$.
Male vaccination could represent also a reliable option for couples undergoing ART cycles because fertility of female partner results yet partially compromised: this could solve the problem linked to sperm banking, avoiding the potential HPV negative effect on sperm quality [52].

The biggest concern of Public Health Programs and clinical practitioners about the cost-effectiveness of HPV male vaccination is linked to the several biases affecting available data. For example, a recent study by Kim and Goldie concluded that the HPV vaccination of all 12-yearold boys would not be cost effective [53]. All readers should realize that this conclusion was obtained despite strong bias: oncologic safety and efficacy in men were not included since it considered only women outcomes; only heterosexual couples were considered without including outcomes in homosexual men; it did not evaluate data about incidence, mortality, and quality of life linked to cancers different from cervix and, finally, it did not contemplate the possible role of HPV male infection on fertility.

\section{Conclusions}

Most of analysed data suggested that the HPV sperm infection could be responsible for a decreased fertility rate through different mechanisms acting at various steps of the human embryo development.

The still debated clinical features related to HPV-DNA sperm infection are the increased risk of early miscarriage and the higher incidence of unexplained male infertility (related to sperm parameters impairment). HPV-DNA sperm test should be realized in semen donors and before ART cycles, despite the limitations linked to in vitro studies evidences. Improvement in the knowledge of HPV-DNA sperm infection mechanisms, timing, and link to fertility impairment could explain most of the actual "idiopathic" male and couple infertility.

The cost-effectiveness analysis related to fertility improvement in HPV vaccinated male requires further evaluation in the next future.

The implications related to possible achievement of herd immunity after a mass population vaccination program could overcome the existing doubts, explaining and resolving the unclear aspects in this field.

\section{Conflict of Interests}

The authors declare that there is no conflict of interests regarding the publication of this paper.

\section{References}

[1] A. R. Giuliano, J.-H. Lee, and W. Fulp, "Incidence and clearance of genital human papillomavirus infection in men (HIM): a cohort study," The Lancet, vol. 377, no. 9782, p. 2006, 2011.

[2] T. S. Patrelli, S. Gizzo, F. Peri et al., "Impact of highly active antiretroviral therapy on the natural history of cervical precancerouslesions: a 17-year institutional longitudinal Cohort Study," Reproductive Sciences. In press. 
[3] S. Gizzo, E. Ancona, T. S. Patrelli et al., "Fertility preservation in young women with cervical cancer: an oncologic dilemma or a new conception of fertility sparing surgery?" Cancer Investigation, vol. 31, no. 3, p. 189, 2013.

[4] C. Saccardi, S. Gizzo, M. Noventa et al., "High-risk human papillomavirus DNA test: could it be useful in low-grade cervical lesion triage? Five-year follow-up," Reproductive Sciences, vol. 21, no. 2, pp. 198-203.

[5] World Health Organization, WHO Laboratory Manual for the Examination of Human Semen and Sperm-Cervical Mucus Interaction, Cambridge, UK, Cambridge University Press, 1999 World Health Organization. Viral cancers, http://www.who.int/vaccine_research/diseases/viral_cancers/ en/print.html.

[6] G. D’Souza, A. R. Kreimer, R. Viscidi et al., "Case-control study of human papillomavirus and oropharyngeal cancer," The New England Journal of Medicine, vol. 356, no. 19, pp. 1944-1956, 2007.

[7] L. Barzon, V. Militello, S. Pagni et al., "Distribution of human papillomavirus types in the anogenital tract of females and males," Journal of Medical Virology, vol. 82, no. 8, pp. 1424-1430, 2010.

[8] J. Doorbar, W. Quint, L. Banks et al., “The biology and life-cycle of human papillomaviruses," Vaccine, vol. 30, supplement 5, pp. F55-F70, 2012.

[9] M. Arbyn, S. de Sanjose, M. Saraiya et al., "EUROGIN 2011 roadmap on prevention and treatment of HPV8 related disease," International Journal of Cancer, vol. 131, no. 9, pp. 1969-1982, 2012.

[10] J. D. Boone, B. K. Erickson, and W. K. Huh, "New insights into cervical cancer screening," Journal of Gynecologic Oncology, vol. 23, no. 4, pp. 282-287, 2012.

[11] T. Malagon, M. Drolet, M. C. Boily et al., "Cross-protective efficacy of two human papillomavirus vaccines: a systematic review and meta-analysis," The Lancet Infectious Diseases, vol. 12, no. 10, pp. 781-789, 2012.

[12] M. Reuschenbach and M. von Knebel Doeberitz, "Diagnostic tests for the detection of human papillomavirus-associated cervical lesions," Current Pharmaceutical Design, vol. 19, no. 8, pp. 1358-1370, 2013.

[13] S. K. Kjaer, C. Munk, J. F. Winther, H. O. Jørgensen, C. J. L. M. Meijer, and A. J. C. Van Den Brule, "Acquisition and persistence of human papillomavirus infection in younger men: a prospective follow-up study among Danish soldiers," Cancer Epidemiology Biomarkers and Prevention, vol. 14, no. 6, pp. 1528-1533, 2005.

[14] M. Lajous, N. Mueller, A. Cruz-Valdéz et al., "Determinants of prevalence, acquisition, and persistence of human papillomavirus in healthy Mexican military men," Cancer Epidemiology Biomarkers and Prevention, vol. 14, no. 7, pp. 1710-1716, 2005.

[15] J. M. Partridge, J. P. Hughes, Q. Feng et al., "Genital human papillomavirus infection in men: incidence and risk factors in a cohort of university students," Journal of Infectious Diseases, vol. 196, no. 8, pp. 1128-1136, 2007.

[16] C. M. Nielson, R. Flores, R. B. Harris et al., "Human papillomavirus prevalence and type distribution in male anogenital sites and semen," Cancer Epidemiology Biomarkers and Prevention, vol. 16, no. 6, pp. 1107-1114, 2007.

[17] A. Garolla, A. Lenzi, G. Pal et al., "Human papillomavirus sperm infection and assisted reproduction: a dangerous hazard with a possible safe solution," Human Reproduction, vol. 27, no. 4, pp. 967-973, 2012.
[18] C. Foresta, A. Garolla, D. Zuccarello et al., "Human papillomavirus found in sperm head of young adult males affects the progressive motility, Fertility and Sterility, vol. 93, no. 3, pp. 802-806, 2010.

[19] J. Pérez-Andino, C. B. Buck, and K. Ribbeck, "Adsorption of human papillomavirus 16 to live human sperm," PLOS ONE, vol. 4, no. 6, Article ID e5847, 2009.

[20] M. A. M. Rintala, S. E. Grénman, P. P. Pöllänen, J. J. O. Suominen, and S. M. Syrjänen, "Detection of high-risk HPV DNA in semen and its association with the quality of semen," International Journal of STD and AIDS, vol. 15, no. 11, pp. 740743, 2004.

[21] D. A. Connelly, P. J. Chan, W. C. Patton, and A. King, "Human sperm deoxyribonucleic acid fragmentation by specific types of papillomavirus," American Journal of Obstetrics and Gynecology, vol. 184, no. 6, pp. 1068-1070, 2001.

[22] C. A. Lee, C. T. F. Huang, A. King, and P. J. Chan, "Differential effects of human papillomavirus DNA types on p53 tumorsuppressor gene apoptosis in sperm," Gynecologic Oncology, vol. 85, no. 3, pp. 511-516, 2002.

[23] M. Cabrera, "Transfection of the inner cell mass and lack of a unique DNA sequence affecting the uptake of exogenous DNA by sperm as shown by dideoxy sequencing analogues," Journal of Assisted Reproduction and Genetics, vol. 14, no. 2, pp. 120-124, 1997.

[24] P. J. Chan, "Sperm-mediated DNA transfer to cells of the uterus and embryo," Molecular Reproduction and Development, vol. 56, supplement 2, pp. 316-318, 2000.

[25] C. Foresta, C. Patassini, A. Bertoldo et al., "Mechanism of human papillomavirus binding to human spermatozoa and fertilizing ability of infected spermatozoa," PLOS ONE, vol. 6, no. 3, Article ID e15036, 2011.

[26] P. L. Hermonat, L. Han, P. J. Wendel et al., "Human papillomavirus is more prevalent in first trimester spontaneously aborted products of conception compared to elective specimens," Virus Genes, vol. 14, no. 1, pp. 13-17, 1997.

[27] M. Matovina, K. Husnjak, N. Milutin, S. Ciglar, and M. Grce, "Possible role of bacterial and viral infections in miscarriages," Fertility and Sterility, vol. 81, no. 3, pp. 662-669, 2004.

[28] A. Dana, K. M. Buchanan, M. A. Goss et al., "Pregnancy outcomes from the pregnancy registry of a human papillomavirus type6/11/16/18 vaccine," Obstetrics and Gynecology, vol. 114, no. 6, pp. 1170-1178, 2009.

[29] M. Skoczynski, A. Gozdzicka-Jozefiak, and A. Kwasniewska, "Prevalence of human papillomavirus in spontaneously aborted products of conception," Acta Obstetricia et Gynecologica Scandinavica, vol. 90, no. 12, pp. 1402-1405, 2011.

[30] A. Perino, L. Giovannelli, R. Schillaci et al., "Human papillomavirus infection in couples undergoing in vitro fertilization procedures: impact on reproductive outcomes," Fertility and Sterility, vol. 95, no. 5, pp. 1845-1848, 2011.

[31] J. E. Brossfield, P. J. Chan, W. C. Patton, and A. King, "Tenacity of exogenous human papillomavirus DNA in sperm washing," Journal of Assisted Reproduction and Genetics, vol. 16, no. 6, pp. 325-328, 1999.

[32] C. Foresta, D. Pizzol, A. Moretti, L. Barzon, G. Pal, and A. Garolla, "Clinical and prognostic significance of human papillomavirus DNA in the sperm or exfoliated cells of infertile patients and subjects with risk factors," Fertility and Sterility, vol. 94, no. 5, pp. 1723-1727, 2010. 
[33] C. Foresta, A. Ferlin, A. Bertoldo, C. Patassini, D. Zuccarello, and A. Garolla, "Human papilloma virus in the sperm cryobank: an emerging problem?" International Journal of Andrology, vol. 34, no. 3, pp. 242-246, 2011.

[34] A. Garolla, D. Pizzol, A. Bertoldo et al., "Association, 1 prevalence, and clearance of human papillomavirus and antisperm antibodies in infected semen samples from infertile patients," Fertility and Sterility, vol. 99, no. 1, pp. 125-131, 2013.

[35] P. J. Chan, B. C. Su, T. Kalugdan, I. M. Seraj, D. R. Tredway, and A. King, "Human papillomavirus gene sequences in washed human sperm deoxyribonucleic acid," Fertility and Sterility, vol. 61, no. 5, pp. 982-985, 1994.

[36] Y. M. Lai, Y. K. Soong, J. F. Lee, F.-P. Yang, H. Y. Huang, and C. C. Pao, "The effect of human papillomavirus infection on sperm cell motility," Fertility and Sterility, vol. 67, no. 6, pp. 1152-1155, 1997.

[37] P. J. Chan, I. M. Seraj, T. H. Kalugdan, and A. King, "Evidence for ease of transmission of human papillomavirus DNA from sperm to cells of the uterus and embryo," Journal of Assisted Reproduction and Genetics, vol. 13, no. 6, pp. 516-519, 1996.

[38] H. You, Y. Liu, M. J. Carey, C. L. Lowery, and P. L. Hermonat, "Defective 3A trophoblast-endometrial cell adhesion and altered $3 \mathrm{~A}$ growth and survival by human papillomavirus type 16 oncogenes," Molecular Cancer Research, vol. 1, no. 1, pp. 25-31, 2002.

[39] J. H. Calinisan, S. R. Chan, A. King, and P. J. Chan, "Human papillomavirus and blastocyst apoptosis," Journal of Assisted Reproduction and Genetics, vol. 19, no. 3, pp. 132-136, 2002.

[40] A. A. Henneberg, W. C. Patton, J. D. Jacobson, and P. J. Chan, "Human papilloma virus DNA exposure and embryo survival is stage-specific," Journal of Assisted Reproduction and Genetics, vol. 23, no. 6, pp. 255-259, 2006.

[41] L. M. Gomez, Y. Ma, C. Ho, C. M. McGrath, D. B. Nelson, and S. Parry, "Placental infection with human papillomavirus is associated with spontaneous preterm delivery," Human Reproduction, vol. 23, no. 3, pp. 709-715, 2008.

[42] L. J. Burkman, "Discrimination between nonhyperactivated and classical hyperactivated motility patterns in human spermatozoa using computerized analysis," Fertility and Sterility, vol. 55, no. 2, pp. 363-371, 1991.

[43] Y. M. Lai, F.-P. Yang, and C. C. Pao, "Human papillomavirus deoxyribonucleic acid and ribonucleic acid in seminal plasma and sperm cells," Fertility and Sterility, vol. 65, no. 5, pp. 10261030, 1996.

[44] F. R. Ochsendorf, "Sexually transmitted infections: impact on male fertility," Andrologia, vol. 40, no. 2, pp. 72-75, 2008.

[45] O. Malhomme, N. Dutheil, M. Rabreau, E. Armbruster-Moraes, J. R. Schlehofer, and T. Dupressoir, "Human genital tissues containing DNA of adeno-associated virus lack DNA sequences of the helper viruses adenovirus, herpes simplex virus or cytomegalovirus but frequently contain human papillomavirus DNA," Journal of General Virology, vol. 78, no. 8, pp. 1957-1962, 1997.

[46] A. R. Giuliano, J.-H. Lee, and W. Fulp, "Incidence and clearance of genital human papillomavirus infection in men (HIM): a cohort study," The Lancet, vol. 377, no. 9769, pp. 932-940, 2011.

[47] S. Gizzo, M. Noventa, and G. B. Nardelli, "Gardasil administration to hr-HPV-positive women and their partners," Trends in Pharmacological Sciences, vol. 34, no. 9, pp. 479-480, 2013.

[48] C. Foresta, D. Pizzol, A. Bertoldo, M. Menegazzo, L. Barzon, and A. Garolla, "Semen washing procedures do not eliminate human papilloma virus sperm infection in infertile patients," Fertility and Sterility, vol. 96, no. 5, pp. 1077-1082, 2011.

[49] M. D. Kaspersen, P. B. Larsen, H. J. Ingerslev et al., "Identification of multiple HPV types on Spermatozoa from human sperm donors," PLoS ONE, vol. 6, no. 3, Article ID e18095, 2011.

[50] L. D. Wise, C. J. Pauley, B. Michael, and J. J. Wolf, "Lack of effects on male fertility from a quadrivalent HPV vaccine in SpragueDawley rats," Birth Defects Research B, vol. 89, no. 5, pp. 376-381, 2010.

[51] R. Berretta, S. Gizzo, A. Dall'asta et al., "Risk of preterm delivery associated with prior treatment of cervical precancerous lesion according to the depth of the cone," Disease Markers, vol. 35, no. 6, pp. 721-726, 2013.

[52] S. Gizzo, E. Ancona, C. Saccardi et al., "Radical trachelectomy: the first step of fertility preservation in young women with cervical cancer," Oncology Reports, vol. 30, no. 6, pp. 2545-2554, 2013.

[53] J. J. Kim and S. J. Goldie, "Cost effectiveness analysis of including boys in a human papillomavirus vaccination programme in the United States," British Medical Journal, vol. 339, Article ID b3884, 2009. 


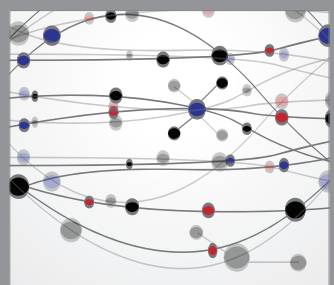

The Scientific World Journal
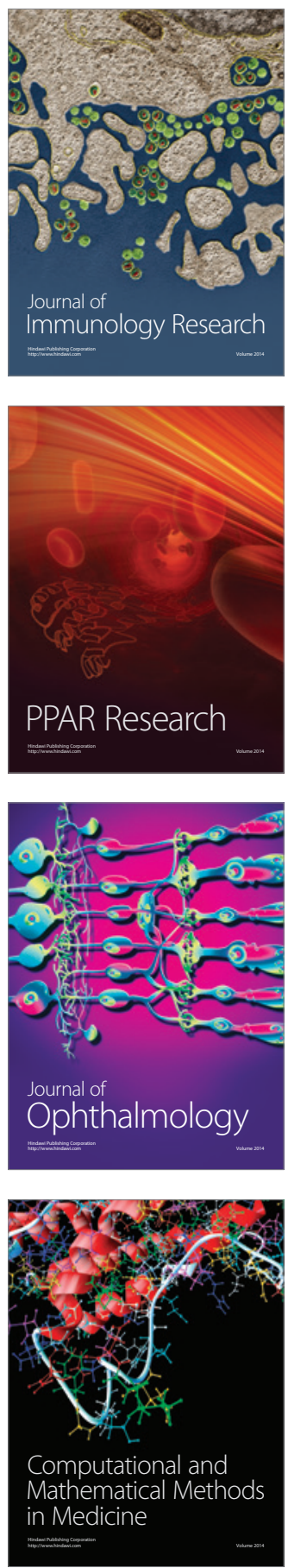

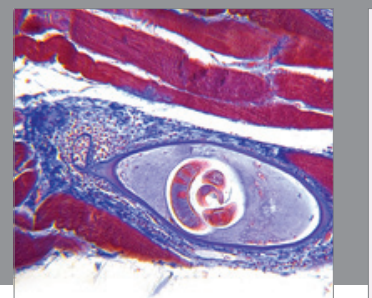

Gastroenterology

Research and Practice
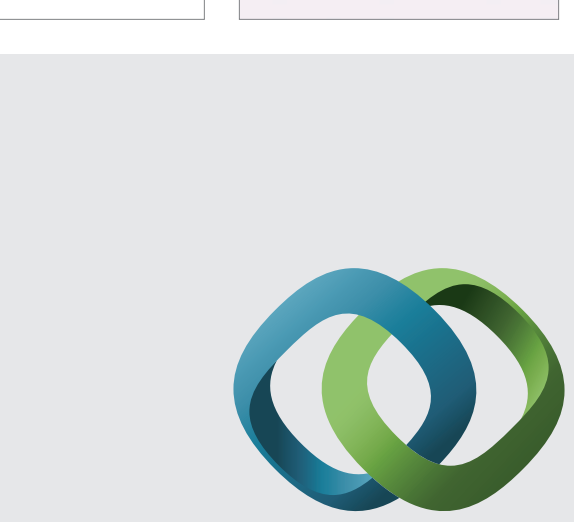

\section{Hindawi}

Submit your manuscripts at

http://www.hindawi.com
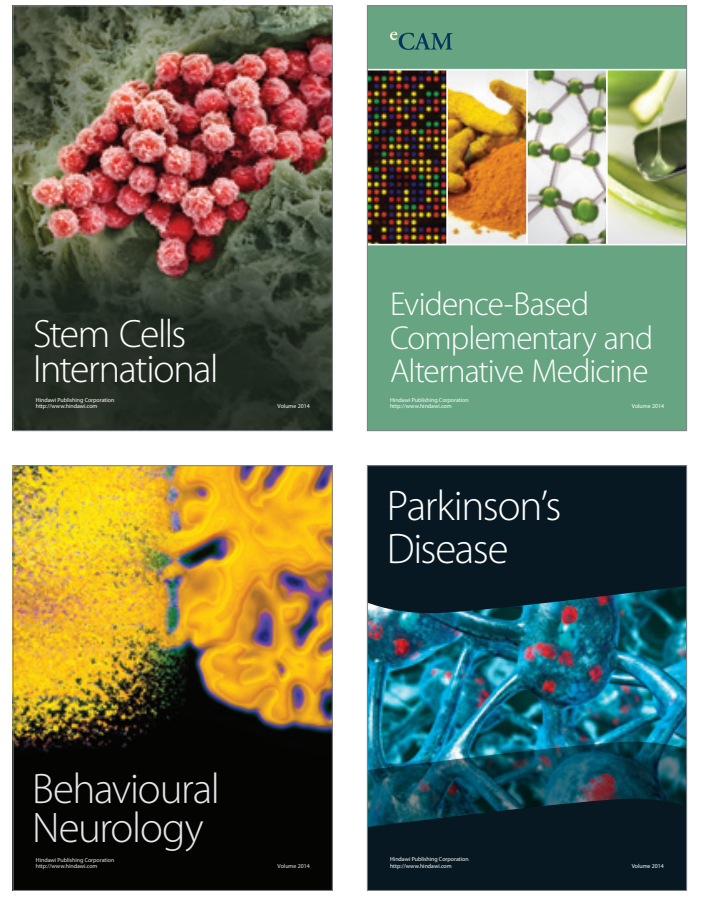
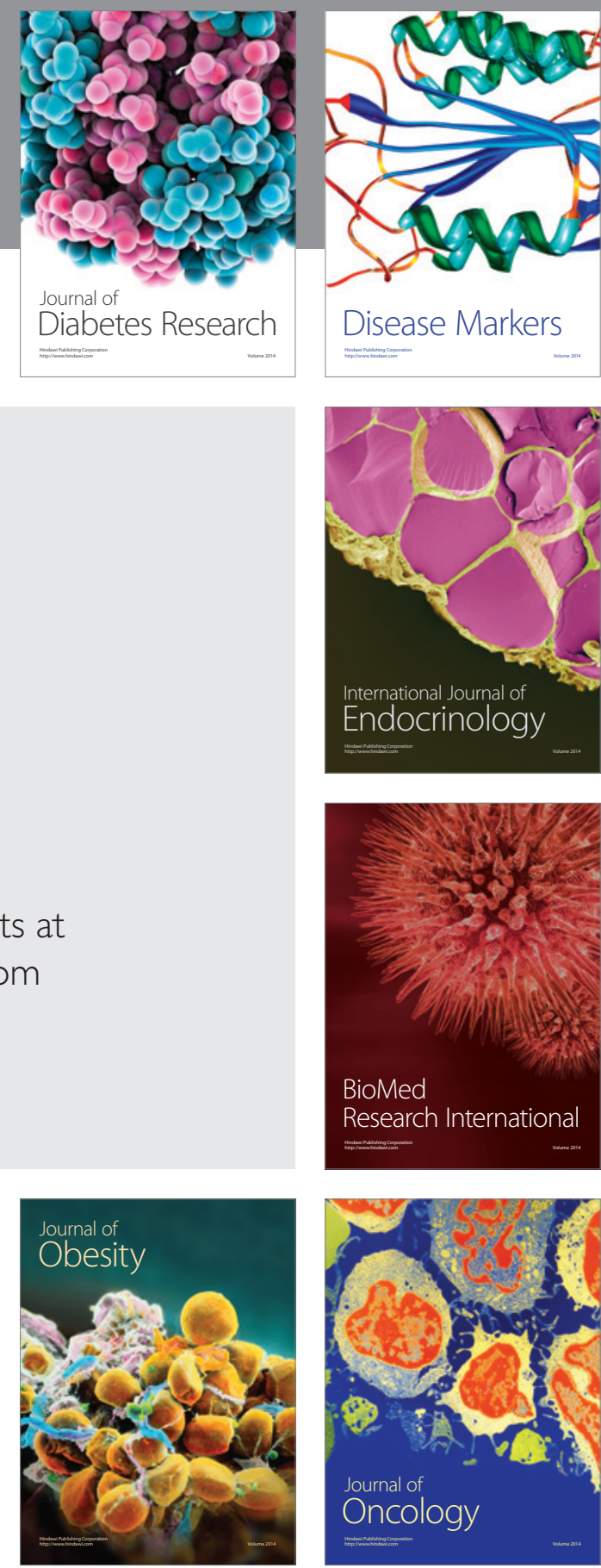

Disease Markers
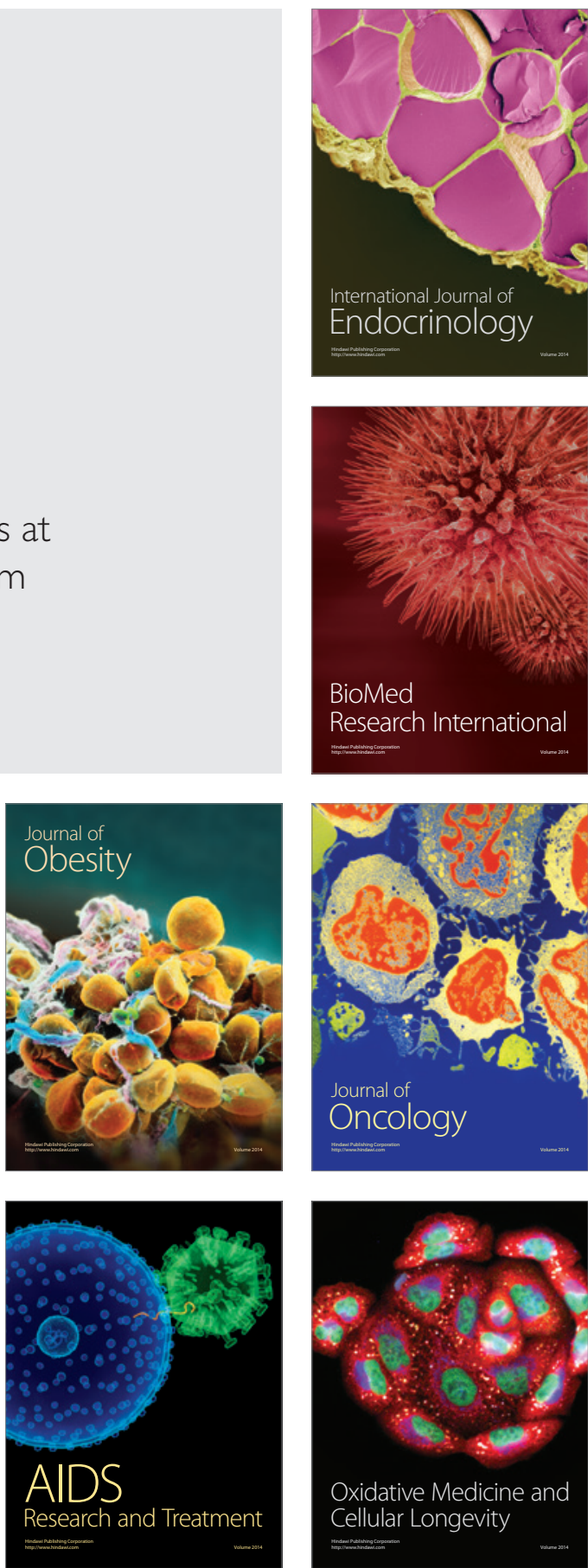